Book Chapter

\title{
Illumination Policies for Stichococcus sp. Cultures in an Optimally Operating Lab- Scale PBR toward the Directed Photosynthetic Production of Desired Products
}

Paraskevi Psachoulia and Christos Chatzidoukas*

Department of Chemical Engineering, Aristotle University of Thessaloniki (AUTH), Greece

*Corresponding Author: Christos Chatzidoukas, Department of Chemical Engineering, Aristotle University of Thessaloniki (AUTH), P.O. Box. 472, 54124 Thessaloniki, Greece

Published June 16, 2021

This Book Chapter is a republication of an article published by Paraskevi Psachoulia and Christos Chatzidoukas at Sustainability in February 2021. (Psachoulia, P.; Chatzidoukas, C. Illumination Policies for Stichococcus sp. Cultures in an Optimally Operating Lab-Scale PBR toward the Directed Photosynthetic Production of Desired Products. Sustainability 2021, 13, 2489. https://doi.org/10.3390/su13052489)

How to cite this book chapter: Paraskevi Psachoulia, Christos Chatzidoukas. Illumination Policies for Stichococcus sp. Cultures in an Optimally Operating Lab-Scale PBR toward the Directed Photosynthetic Production of Desired Products. In: Maria Helena Henriques, editor. Prime Archives in Sustainability. Hyderabad, India: Vide Leaf. 2021.

(C) The Author(s) 2021. This article is distributed under the terms of the Creative Commons Attribution 4.0 International License(http://creativecommons.org/licenses/by/4.0/), which 
permits unrestricted use, distribution, and reproduction in any medium, provided the original work is properly cited.

Author Contributions: P.P. wrote and edited the manuscript, performed and validated the experiments, and collected the data. C.C. wrote and reviewed the manuscript, supervised the experimental work, and conceptualized the project. All authors have read and agreed to the published version of the manuscript.

Funding: This research has been co-financed by the European Union and Greek national funds through the Operational Program Competitiveness, Entrepreneurship and Innovation, under the call RESEARCH-CREATE-INNOVATE (project code: T2EDK-02279). Project title: Human nutrition, animal and fish feeding on microalgae derived products through sustainable photosynthetic autotrophic cultures.

Data Availability Statement: Data is contained within the article

Acknowledgments: The authors would like to thank the Biochemical Engineering and Environmental Biotechnology laboratory of the Department of Environmental Engineering, Technical University of Crete, for kindly supplying the employed microalgal strain. (Stichococcus sp.)

Conflicts of Interest: The authors declare no conflict of interest.

\section{Abstract}

The light spectrum effect on the cultivation efficiency of the microalgae strain Stichococcus sp. is explored, as a means of potentially intensifying the biomass productivity and regulating the cellular composition. Stichococcus sp. batch culture experiments, within a $3 \mathrm{~L}$ bench-top photobioreactor (PBR), are designed and implemented under different light spectrum profiles (i.e., cool white light (WL), cool white combined with red light (WRL), and cool white combined with blue light, (WBL)). The obtained results indicate that the studied strain is capable of adapting its metabolite profile to the light field to 
which it is exposed. The highest biomass concentration $(3.5 \mathrm{~g} / \mathrm{L})$, combined with intense carbohydrate accumulation activity, resulting in a respective final concentration of $1.15 \mathrm{~g} / \mathrm{L}$ was achieved within 17 days using exclusively cool white light of increasing intensity. The addition of blue light emitting diodes (LED) light, combined with appropriately selected culture conditions, contributed significantly to the massive synthesis and accumulation of lipids, resulting in a concentration of $1.43 \mathrm{~g} / \mathrm{L}$ and a respective content of $46.13 \% \mathrm{w} / \mathrm{w}$, with a distinct impact on biomass, carbohydrates and proteins productivity. Finally, a beneficial contribution of red LED light to the protein synthesis is recognized and this can be conditionally amplified provided nitrogen sufficiency in the culture medium.

\section{Keywords}

Microalgae Biomass Composition; Blue LED Light; Red LED Light; Lipids;

Proteins;

Carbohydrates; Biorefinery

\section{Introduction}

Microalgae have recently attracted noticeable research interest as a potential renewable source for several value-added products synthesis, including carbohydrates, proteins, lipids, and pigments. Due to their great diversity, better photosynthetic efficiency than higher plants, and low nutrient requirements, microalgae hold great promise for the co-production of highvalue compounds, potentially used in a variety of biotechnological and commercial applications (pharmaceuticals, nutraceuticals, cosmetics, biofuels, animal feed, aquaculture, etc.). Moreover, due to their incomparable photosynthetically induced carbon assimilation rates and their high intracellular accumulation rates of lipids, microalgae carry enormous potential to contribute to a clean-energy future. In particular, microalgal biodiesel, a third-generation renewable biofuel, has recently received commercial interest, since, without overlooking the management practices for its environmentally sustainable production [1], it presents some distinct advantages, including renewability, non-toxicity, and environmental 
friendliness $[2,3]$. However, it has become evident that with the current state-of-the-art technologies, a microalgae-based biodiesel production process is only conditional sustainable, and its economic feasibility is ensured only upon the adoption of the biorefinery concept as an operational strategy [4]. The biorefinery concept is tightly related to the multiple-commodity production approach and exploitation of all biomass components simultaneously for commercial applications and energy recovery. However, such a production scheme requires understanding the biochemistry of the carbon storage metabolism in microalgae cells. Only then, and through a systematic handling of the cultivation process operating profile, will the efficient steering of microalgae cultures toward the overproduction of the desired products and sufficient improvement of the process productivity and ultimate economic viability potential be enabled [5].

Both microalgae culture growth and their photosynthetic metabolite production rates are affected by many environmental factors, such as nutrient type and concentration [6,7], $\mathrm{CO}_{2}$ availability $[8,9]$, temperature $[10,11]$, and light (wavelength, intensity, and photoperiod) [12-14]. Many studies identify that under intensively or moderately stressed conditions, such as nutrient limitation/deprivation, increased/decreased light intensity, or high salt concentrations, microalgae cells develop survival strategies and undergo morphological and biochemical changes $[15,16]$.

Light quality, in terms of both wavelength and intensity, represents one of the most important parameters for phototrophic microalgae systems, as it is the driving force for the photosynthesis and the regulation of several cellular processes. Light emitting diodes (LEDs), capable of producing monochromatic light, can be successfully used for microalgae cultivation to efficiently control the productivity of desirable intracellular metabolites. Photosynthetically active radiation (PAR) is the wavelength range between 400 and $700 \mathrm{~nm}$ that sufficiently supports the energy requirements of the photosynthesis process $[17,18]$. Photosynthesis is initiated when light is captured by light-harvesting antenna complexes 
composed of different light-capturing pigments [19]. Chlorophylls ( $\mathrm{a}$ and $\mathrm{b}$ ) are the primary pigments essential for oxygenic photosynthesis in green algae, delivering the energy from the light photons to the molecules in the form of reducing potential, whereas phycobilins and carotenoids are accessory pigments. Carotenoids, in particular, regulate the protection of the photosynthetic apparatus, while phycobilins contribute to light harvesting by increasing the absorption spectrum of microalgal cells in low light conditions $[17,19]$. These pigments exhibit a characteristic color, as they absorb light in specific wavelengths (Table 1). The use of light wavelengths that correspond to the two major absorption bands of chlorophyll a and $\mathrm{b}$, blue (430-475 $\mathrm{nm}$ ) and red (630-680 nm) [20,21], has recently received widespread attention for potentially enhancing the photosynthesis process in microalgae cultures $[17,18]$.

Table 1: Ranges of the absorption bands of the major pigments in green microalgae and the employed light sources.

\begin{tabular}{|l|l|l|}
\hline Pigment Group & Colour & $\begin{array}{l}\text { Absorption } \\
\text { Bands }\end{array}$ \\
\hline Chlorophyll a, b & Green & $\begin{array}{l}430-475 \\
630-680\end{array}$ \\
\hline Phycobilins & Blue, red & $500-650$ \\
\hline Carotenoids & Yellow, orange & $400-550$ \\
\hline & Light Sources Used & \\
\hline White LED Light & White & $\begin{array}{l}400-700 \\
\text { (peak at 450) }\end{array}$ \\
\hline Blue LED strip & Blue & 460 \\
\hline Red LED strip & Red & 630 \\
\hline
\end{tabular}

Indeed, many experimental results indicate that blue and red light not only promote the photosynthetic rate when compared to white, but also cause variations to the regulation of several cellular metabolites and morphogenesis in algae. However, different conclusions about the influence of light wavelength on microalgae culture performance have been reported. Teo et al. (2014) claimed that blue light enhanced the culture growth and oil productivity for both Tetraselmis sp. and Nannochloropsis sp. when compared to red, red-blue, and white light [22]. Similar results have been obtained by Das et al. (2011), who concluded that the use of blue light favored the biomass productivity of 
both mixotrophic and autotrophic cultures of Nannochloropsis sp. when compared to other types of light (i.e., red, green, and white) [23]. On the other hand, the use of red light as a tool to enhance the photosynthetic process has been advocated in several studies. When Chlorella vulgaris was cultivated under white, red, green, and blue light, it exhibited the maximum growth rate when exposed to red light [24]. Shu et al. (2011) also reported that both monocultures of Chlorella sp. and mixed cultures with Saccharomyces cerevisiae attained greater specific growth rates under red LED light [25]. Kim et al. (2014) noticed that exposure of $C$. vulgaris cultures to blue light led to significantly increased cell size, whereas red light resulted in a maximum number of smaller cells [14]. The same observations were recorded by Oldenhof et al. (2006), who pointed out that when Chlamydomonas reinhardtii cultures were subjected to blue light, cells were continuously growing for a longer period and attained larger sizes than under red light [26]. Similar results were obtained by Koc et al. (2013), who found that Chlorella kessleri cells grown under blue LED light were larger than those grown under red or white fluorescent light, while red light resulted in higher biomass concentration than blue LED light, even though the average cell size was smaller [27]. In a study by Rendón et al. (2013), who tried to elucidate the effect of $\mathrm{CO}_{2}$ supply and illumination of $C$. vulgaris cultures at different light wavelengths, the highest biomass production was found when algal cultures were supplied with $8.5 \% \quad \mathrm{CO}_{2}$ and exposed to white light [28]. Furthermore, the use of white light was found to increase the production rate of Scenedesmus sp. when compared to blue, green, and red light. Nonetheless, in the same study, when red and blue LED light were combined in different intensity ratios, remarkably higher biomass production rates were measured than with a single wavelength of light (even white light), irrespective of the ratio used to combine the light wavelengths [29]. Abiusi et al. (2014) presented a complete study over the effect of different light wavelengths on cell size and cell cycle, growth rate, productivity, photosynthetic efficiency, and biomass composition of Tetraselmis suecica. Whereas red and white light were the most effective regarding biomass productivity and photosynthetic efficiency, cultivation under red light attained the highest protein content, while 
cultures grown under blue and green light exhibited the highest lipid and carbohydrate content, respectively. This study also confirmed the suggestion that red light on microalgae cultures could lead to smaller cells [30]. Finally, Mutaf et al. (2019), who recently investigated the effect of different culture media and different light policies on the cultivation of Stichococcus bacillaris, reported that even if blue and red light did not play a critical role on the culture growth kinetics, the use of these two lights had a significant impact on chlorophyll accumulation and lipid profile [31].

From the numerous studies surveyed, it was unambiguously concluded that the effect of the light on microalgae cell growth and preferred expression of desirable metabolites is most likely species- and culture condition-dependent, and currently the gained knowledge is still insufficient as a general pattern cannot be described yet. This might be due to the fact that very few studies examine microalgae cultures at the scale of a photobioreactor under well monitored and controlled conditions. On the other hand, the majority focus only on total microalgae biomass production and specific intracellular metabolites concentration, especially lipids, neglecting the effect of different light wavelengths on the synthesis of other, equally important and commercially exploitable cell products (proteins, carbohydrates, and pigments). The knowledge of total bioproducts accumulation rates is essential for screening the light spectrum effect on the cultivation of a specific microalgae strain and extracting significant conclusions about the impact of light wavelength from the biorefinery perspective.

In our previous study, the effect of five process variables (i.e., illumination flux, aeration rate, $\mathrm{CO}_{2}$ supply rate, nitrogen concentration, and salinity) on the performance of Stichococcus sp. cultures was thoroughly investigated [32]. Precisely, employing the Taguchi design experiment method, the biomass production rate and the accumulation capacity of the microalgae population in carbohydrates, proteins, and lipids were correlated with the above key process variables, and the best operating policy that selectively maximizes the component of interest was explored. With this experimental background over the strain 
Stichococcus sp., the aim of the present work is to quantitatively examine the effect of the light spectrum on the growth and bioproducts concentration (lipids, proteins, and carbohydrates) of the studied marine microalgae strain. To our knowledge, the influence of light spectrum on the growth and complete biochemical composition on the cultivation of Stichococcus sp. in the scale of a photobioreactor system is elucidated for the first time. Thus, Stichococcus sp. cultures are exposed to different combinations of cool white, red, and blue LED light in a labscale photobioreactor (PBR), with simultaneous re-adjustment of the already explored optimal operational variables, to unravel the potential of employing the proposed cell factories in a biorefinery plant.

\section{Materials and Methods Microalgae Strain and Pre-Culture Conditions}

The marine strain Stichococcus sp. (identified with the 18SrDNA gene sequence analysis) was isolated from the Crete coastal area in Southern Greece. Stichococcus is a genus of green algae (Chlorophyta) characterized by a simple morphology, with cell size ranging from 2 to $6 \mu \mathrm{m}$, organized in filamentous or unicellular structures [33], with some species (e.g., S. bacillaris) being distinguished for their resistance to temperature, salinity and $\mathrm{pH}$ variation, their lipid content, as well as their capacity to remove efficiently heavy metals from wastewater [32,34-36].

Both for the stock cultures and the preparation of pre-cultures inoculating the bioreactor, cells were cultivated in Erlenmeyer flasks of $500 \mathrm{~mL}$ capacity with a working volume of $300 \mathrm{~mL}$ and incubated for 15 days at $25{ }^{\circ} \mathrm{C}$ in a shaking incubator (GFL 3031), at a constant shaking rate of $80 \mathrm{rpm}$. Pre-cultures were regenerated every 15 days in new flasks by inoculating fresh medium with the 15-day-old pre-culture at a volume ratio of approximately $1 / 20$ to let the new pre-culture start with initial $\mathrm{OD}_{600 \mathrm{~nm}}$ around 0.25 . Thus, it was guaranteed that all the bioreactors were inoculated with pre-culture cells of roughly the same history. Modified Bold Basal medium with a 3-fold increase of its nitrogen content and vitamin addition $(3 \mathrm{~N}-\mathrm{BBM}+\mathrm{V})$ was used both for pre-cultures and stock cultures. 
Precisely, the medium contained nutrients (in $\mathrm{g} / \mathrm{L}$ ): $\mathrm{NaNO}_{3} 0.75$, $\mathrm{KH}_{2} \mathrm{PO}_{4} 0.175, \mathrm{~K}_{2} \mathrm{HPO}_{4} 0.075, \mathrm{MgSO}_{4} .7 \mathrm{H}_{2} \mathrm{O} 0.075, \mathrm{CaCl}_{2} .2 \mathrm{H}_{2} \mathrm{O}$ 0.025, $\mathrm{NaCl}$ 0.025; trace elements (in $\mathrm{mg} / \mathrm{L}$ ): $\mathrm{FeSO}_{4} \cdot 7 \mathrm{H}_{2} \mathrm{O} 0.60$, $\mathrm{Na}_{2}$ EDTA 3.92, $\mathrm{Na}_{2} \mathrm{MoO}_{4} .2 \mathrm{H}_{2} \mathrm{O} \quad 0.03, \quad \mathrm{CoCl} .6 \mathrm{H}_{2} \mathrm{O} \quad 0.02$, $\mathrm{MnCl}_{2} \cdot 4 \mathrm{H}_{2} \mathrm{O} \quad 0.25, \mathrm{ZnCl}_{2} \quad 0.03$; and vitamins (in $\mathrm{mg} / \mathrm{L}$ ): Thiamine-B1 1.2, Cobalamin-B12 0.01. All nutrients were autoclaved at $121{ }^{\circ} \mathrm{C}$ for $20 \mathrm{~min}$ in separate solutions. The trace elements and vitamin solutions were filter-sterilized using filters (Whatman Polytetrafluoroethylene- PTFE- syringe filters, 0.2 $\mu \mathrm{m})$. The medium initial $\mathrm{pH}$ value was equal to 6.5 and was regulated by the phosphate buffer, already contained in the culture medium (i.e., $\mathrm{KH}_{2} \mathrm{PO}_{4} / \mathrm{K}_{2} \mathrm{HPO}_{4}$ ). Atmospheric air was sparged into the liquid medium through a filter with $0.20 \mu \mathrm{m}$ pore size (Whatman PTFE syringe filters, $0.2 \mu \mathrm{m}$ ) at a flow rate of $0.2 \mathrm{~L} / \mathrm{min}$ to ensure sufficient aeration. For the illumination of cultures, fluorescence lamps with warm daylight $3000 \mathrm{~K}(6500$ $\mathrm{lm}$ ) were used. A photoperiod of $16 \mathrm{~h}$ lighting followed by $8 \mathrm{~h}$ darkness was applied.

\section{PBR Experiments}

The experiments of Stichococcus sp. cultures implemented in the present study to explore the synergistic role of different light spectrums in regulating either the culture growth rate or cell composition were performed in a lab-scale bench-top photobioreactor (FerMac 320, Electrolab Biotech Limited, UK), with a total capacity of $3 \mathrm{~L}$. The research outcome of a preliminary study [32], published by the same research group, was employed to determine the appropriate PBR operating profile. More specifically, given that the culture aeration rate, $\mathrm{CO}_{2}$ supply rate, and initial nitrogen concentration in the culture medium were distinguished as the most important operating factors and examined for their contribution to the total variance of biomass and products concentration, their optimal values found in that work were adopted in the present study. Thus, in all the PBR experiments, separate feeding streams of atmospheric air and $\mathrm{CO}_{2}$ (purity $\geq 99.9 \%$ ) at a constant flow rate of $0.1 \mathrm{~L} / \mathrm{min}$ and $2.5 \mathrm{~mL} / \mathrm{min}$, respectively, were filtered (Whatman PTFE filters, $0.2 \mu \mathrm{m}$ ) and sparged into the photobioreactor containing 2 $\mathrm{L}$ of medium (including the inoculum). The decision of the PBR 
agitation rate and intensity of the incident light is discussed separately below since these operating values were dynamically adjusted with the progress of the culture growth. The composition of the medium used for all the experiments in the PBR was the same as that used for the pre-cultures.

For the $\mathrm{pH}$ control, the $\mathrm{PBR}$ was equipped with a $\mathrm{pH}$ measuring electrode (F-695-B225-DK, Broadley-James, Irvine CA, USA) immersed in the culture; automated addition of buffer solutions of $\mathrm{NaOH}(1 \mathrm{M})$ and $\mathrm{HCl}(1 \mathrm{M})$ was implemented by peristaltic pumps holding the $\mathrm{pH}$ at the set point value of 6.7 with a deadband of 0.1. The dissolved oxygen concentration (D.O.) in the culture medium was also measured with a suitable selective electrode (D140-B120-PT-D9, Broadley-James, Irvine CA, USA) and expressed as a percentage of the respective saturation value of the medium prior to the PBR inoculation. A spherical sensor (US-SQS Spherical Micro Quantum Sensor,Heinz Walz $\mathrm{GmbH}$, Effeltrich, Germany), submerged inside the culture at a fixed point, was used for light intensity measurement. Temperature was maintained at $25 \pm 0.1{ }^{\circ} \mathrm{C}$ using a bioreactor cooling-heating system, i.e., chilled water circuit via a cooling coil and heating via heater mat. The photoperiod was set to $16: 8$ $\mathrm{h}$ light/darkness. The outflow gas stream from the top of the bioreactor was directed through a gas analyzer (Model 902P $\mathrm{O}_{2} / \mathrm{CO}_{2}$ Analyser, Quantek Instruments, Grafton MA, USA) to evaluate the $\mathrm{CO}_{2}$ quantity consumed and $\mathrm{O}_{2}$ produced, as an indication of the photosynthetic efficiency of the culture. A sufficient pre-culture volume was used to inoculate the PBR, resulting in initial OD $@ 600 \mathrm{~nm}$ and DCW (Dry Cell Weight) values of 0.25 and $0.03(\mathrm{~g} / \mathrm{L})$, respectively.

\section{Analytical Measurements}

Frequent sampling and off-line analysis was applied to monitor the overall progress of the Stichococcus sp. cultures. Precisely, samples of 50-35 mL of culture broth were collected at 24-h intervals to perform measurements of both culture growth rate and accumulation rate of the products of interest. Cell growth, measured as optical density (OD) at $600 \mathrm{~nm}$ in a UV-Vis spectrophotometer (Lamda 35, Perkin Elmer, Akron OH, USA), 
was assessed twice a day (once immediately after the completion of the dark period and then $8 \mathrm{~h}$ after the beginning of the illumination period) to provide accurate growth monitoring. Determination of dry biomass concentration, measured as dry cell weight (DCW), was carried out by filtering $5 \mathrm{~mL}$ of culture through a pre-weighted glass microfiber filter (Whatman 934$\mathrm{AH}$, pore diameter $0.2 \mu \mathrm{m}$ ), then dried at $50{ }^{\circ} \mathrm{C}$ overnight, and finally weighted at a high precision micro-balance (XP 105, Mettler Toledo, Columbus OH, USA). Microscopic observations of the culture were carried out daily in an optical microscope (DM 2000, Leika, Wetzlar, Germany).

For the determination of chlorophylls (a) and (b) concentration, 2 $\mathrm{mL}$ samples of the culture were centrifuged and the precipitate was washed with distilled water three times. In total, $2 \mathrm{~mL}$ of pure methanol (99.8\%) was then added to the cell pellet and the mixture was vortexed and left in dark at room temperature for 20 min. The amount of pigments was calculated (in $\mathrm{mg} / \mathrm{L}$ ) via Equations (1) and (2), with prior measurement of the supernatant absorbance at 652 and $665 \mathrm{~nm}$ in a UV-Vis spectrophotometer (Lamda 35, Perkin Elmer, Akron OH, USA) [37,38]:

Chlorophyll $\mathrm{a}=16.72 \cdot \mathrm{A}_{@ 665}-9.16 \cdot \mathrm{A}_{@ 652}$

Chlorophyll $\mathrm{b}=34.09 \cdot \mathrm{A}_{@ 652}-15.28 \cdot \mathrm{A}_{@ 665}$

Lipids contained in microalgae cells were extracted, and lipid content was determined gravimetrically according to the Folch extraction protocol [39]. More specifically, a sufficient quantity of culture broth was centrifuged at $7000 \times \mathrm{g}$, and cell pellets were frozen at $-20{ }^{\circ} \mathrm{C}$ and then lyophilized. In total, $2 \mathrm{mg}$ of the freeze-dried algal biomass were treated with $1.5 \mathrm{~mL}$ of chloroform-methanol $(2: 1 \mathrm{v} / \mathrm{v})$ solvent mixture and underwent sonication within an ice-bath for $15 \mathrm{~min}$, at $50 \%$ of the sonicator's maximum amplitude (Vibra Cell VC-505, Sonics \& Materials, Newtown CT, USA). The solution was centrifuged and the solvent was collected. The procedure was repeated three times with the submerged biomass to ensure total lipids extraction. Then, in the liquid solvent phase, an aqueous solution of $\mathrm{KCl} 0.88 \%(\mathrm{w} / \mathrm{v})$ of volume equal to $20 \%$ of the final solvent volume was added, resulting in the formation of two phases. The 
upper phase was gently removed and $0.3 \mathrm{~mL}$ Pure Solvents Upper Phase mixture [39] was added to the lower phase. After complete separation of the two phases, the upper phase was removed, and this step was repeated to ensure satisfactory washing of the crude extract. A sufficient amount of methanol was then added to the lower phase up to a final total volume of $4.5 \mathrm{~mL}$. The derived single-phase mixture containing the extracted lipids was collected and the lipids dried overnight at 45 ${ }^{\circ} \mathrm{C}$ and were weighed with a precision microbalance (XP 105, Mettler Toledo, Columbus OH, USA).

Proteins were extracted from $2 \mathrm{mg}$ freeze-dried biomass upon treatment with $9.6 \mathrm{~mL}$ of aqueous solution of $\mathrm{NaOH}(0.5 \mathrm{M})$ containing 5\% methanol (in volume fraction) and $0.4 \mathrm{~mL}$ phosphate buffer $(0.05 \mathrm{M})$ and sonication in an ice-bath. The cell suspension was homogenized for $10 \mathrm{~min}$ at $50 \%$ of the sonicator's maximum amplitude (Vibra Cell VC-505, Sonics \& Materials, Newtown CT, USA) to ensure cell breakage and protein release following the applied protocol [40]. After homogenization, $5 \mathrm{~mL}$ of the aqueous solution $(\mathrm{NaOH} 0.5 \mathrm{M}$, $5 \% \mathrm{v} / \mathrm{v} \mathrm{MeOH}$ ) was added and samples were heated at $100{ }^{\circ} \mathrm{C}$ for $30 \mathrm{~min}$ under continuous stirring. The protein content was measured with the aid of a Micro-BCA kit (Thermo Scientific, Waltham MA, USA) at a microplate spectrophotometer (ELx808 Microplate Reader, BioTek, Winooski VT, USA) [41]. The calibration curve was obtained with Bovine Serum Albumin (BSA) solutions of known concentrations.

For the measurement of the carbohydrates content, $2 \mathrm{mg}$ of lyophilized algal biomass was redispersed in $1 \mathrm{~mL} \mathrm{HCl}$ solution $(2.5 \mathrm{M})$ and subsequently incubated at $100{ }^{\circ} \mathrm{C}$ for $3 \mathrm{~h}$ under continuous stirring to achieve cell membrane breakage and reduction of polysaccharides, oligosaccharides, and disaccharides to monosaccharides. After neutralization $(2.5 \mathrm{M}$ $\mathrm{NaOH}$ ) and centrifugation, the phenol-sulfuric method was used to quantify the neutral monomeric sugar content as glucose equivalent by treating the unknown samples with $1 \mathrm{~mL}$ phenol solution $1 \%(\mathrm{w} / \mathrm{v})$ and $5 \mathrm{~mL} \mathrm{H}_{2} \mathrm{SO}_{4} 96 \%(\mathrm{w} / \mathrm{w})$ and measuring the absorption at $483 \mathrm{~nm}$ (Lamda 35, Perkin Elmer, Akron OH, USA) [42]. Solutions of D-glucose of known concentrations 
were used as reference standards for calculating the glucose concentration-absorption calibration curve.

Finally, the nitrate concentration at the culture medium was determined by measuring the absorbance of the supernatant at $220 \mathrm{~nm}$ using a UV-vis spectrophotometer (Lamda 35, Perkin Elmer, Akron OH, USA) [43].

\section{Illumination Profile and Agitation Rate}

Microalgae cultivation systems are strongly dependent on light availability. Supposing optimal temperature, sufficient nutrients, and $\mathrm{CO}_{2}$ supply, limitation to the photosynthesis rate and thus biomass productivity falls to light insufficiency [19]. There is a strong need to evaluate the potential of maximizing light utilization by microalgae to enhance their performance. One serious concern on continuous stirred tank photobioreactor units used for photoautotrophic microalgae cultures arises from the realization of light exponential attenuation upon its radial penetration from the surficial culture layer to the internal ones. As photons impinge on the reactor surface, they are absorbed by cells, scattered, and reflected, resulting in a decrease in light intensity as the distance from the PBR surface increases. Constant PBR illumination may lead to a reduction of biomass productivity, as the outermost layer of cells in the bioreactor are exposed to excessive intensity, resulting in photo-inhibition, whereas inadequate light intensity in the innermost layer of the culture, because of cells' self-shading, becomes growth-limiting. For given geometric characteristics of bioreactor vessels, Carvalho et al. (2011) emphasized the need for intensive stirring in PBRs to increase the efficiency of the light usage by microalgae cells and to attain a uniform exposure of the microalgae cell population to the incident light [16].

In the present study, different light spectrum profiles were investigated to potentially induce controlled biomass compositional changes of the marine strain Stichococcus sp. The lighting policies were implemented under predefined optimal culture conditions and heuristically adjusted agitation and incident light flux profiles. A custom-made lighting box, equipped with 19 cool white daylight LED lamps (Osram SubstiTube advanced, $10 \mathrm{~W} / 6500 \mathrm{~K}, 1100 \mathrm{~lm})$ placed 
symmetrically at its internal sides, was employed for the photobioreactor illumination in all the experiments. Five stripes of blue and five stripes of red LED light with a total length and intensity of $10.5 \mathrm{~m}$ and $75.6 \mathrm{~W}$ for each color were also amended in this structure, as shown in Figure 1a and Figure 1b. The emission spectrum of the light sources used in the present study, recorded via a mini-spectrometer (RC-VIS: C9407, Hamamatsu, Japan), are depicted in Figure 2a and Figure 2b. The employed white LED lamps produce a light spectrum with a sharp peak at $450 \mathrm{~nm}$ (blue light) and a broader distribution at the rest of the visible spectrum, thus emitting a cool white light. On the other hand, red and blue LED strips emit exclusively at the red and blue region of the light spectrum, with a sharp peak at $630 \mathrm{~nm}$ and $460 \mathrm{~nm}$, respectively.

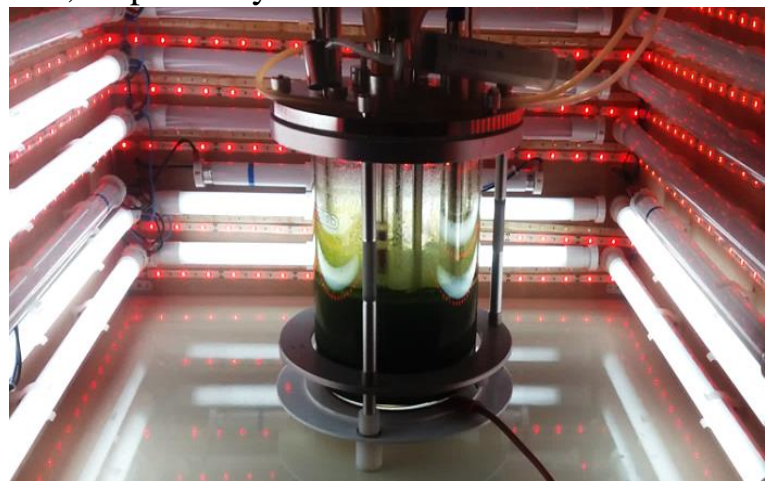

(a)

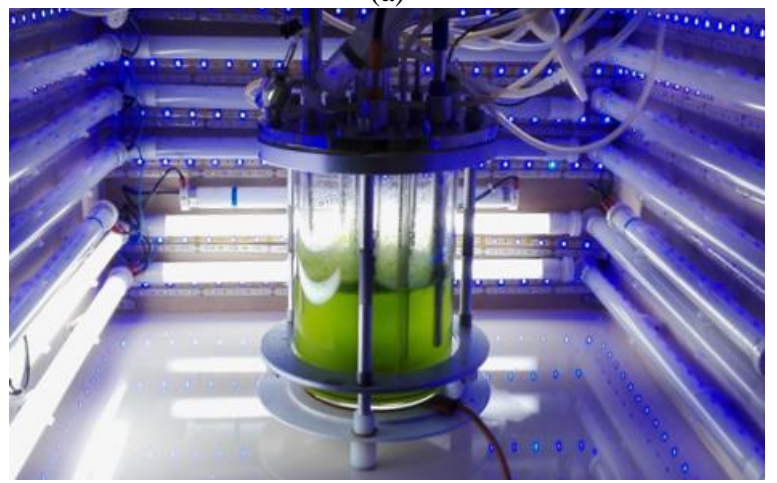

(b)

Figure 1: Experimental setup for the cool white light (WL), cool white combined with red light (WRL), and cool white combined with blue light (WBL) experiments (illumination during the WRL (a) and WBL (b) experiments is shown). 


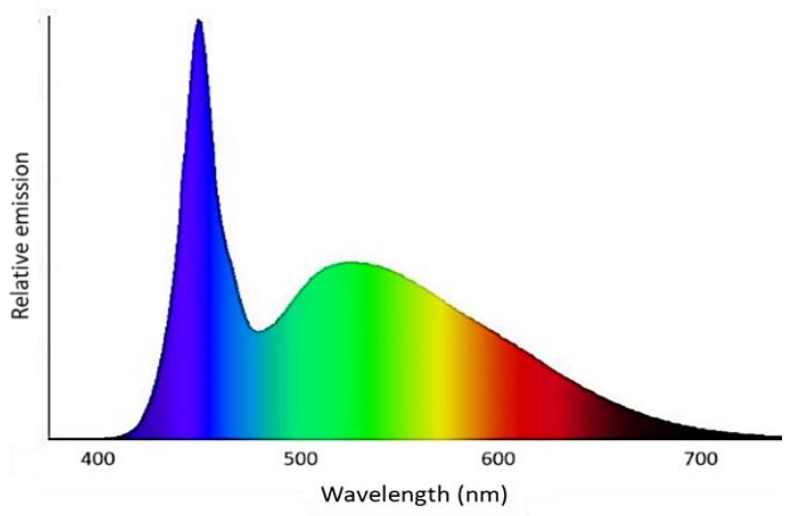

(a)

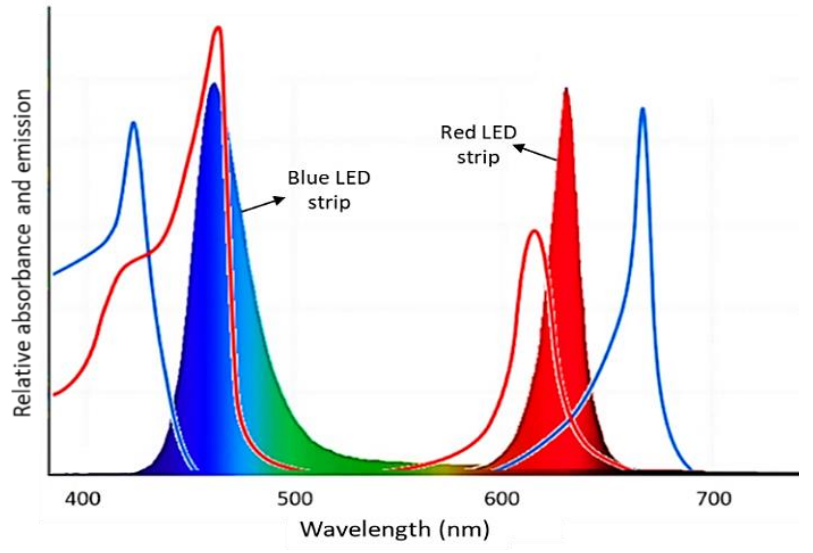

(b)

Figure 2: Spectral distribution of cool white light emitting diodes (LED) light (a), and absorption spectra of chlorophyll a (blue line) and chlorophyll b (red line) compared to emission spectra of a Red LED strip and Blue LED strip (b).

An innovative approach was adopted to mitigate the constraint of light saturation. Precisely, a time-escalating profile of illumination (4400-20,900 lm) was applied in all experiments during different culture stages by manually turning on additional lamps symmetrically around the bioreactor, in combination with a gradual increase of agitation in a range of $100-320 \mathrm{rpm}$. More specifically, in all experiments the initial culture agitation rate was selected equal to $100 \mathrm{rpm}$. This was increased to $150 \mathrm{rpm}$ on 
the third day of the cultivation period, while from the fourth to the twelfth day it was increased in a stepwise mode by $10 \mathrm{rpm}$ every day and by $20 \mathrm{rpm}$ from the thirteenth to the sixteenth day (Figure 3).

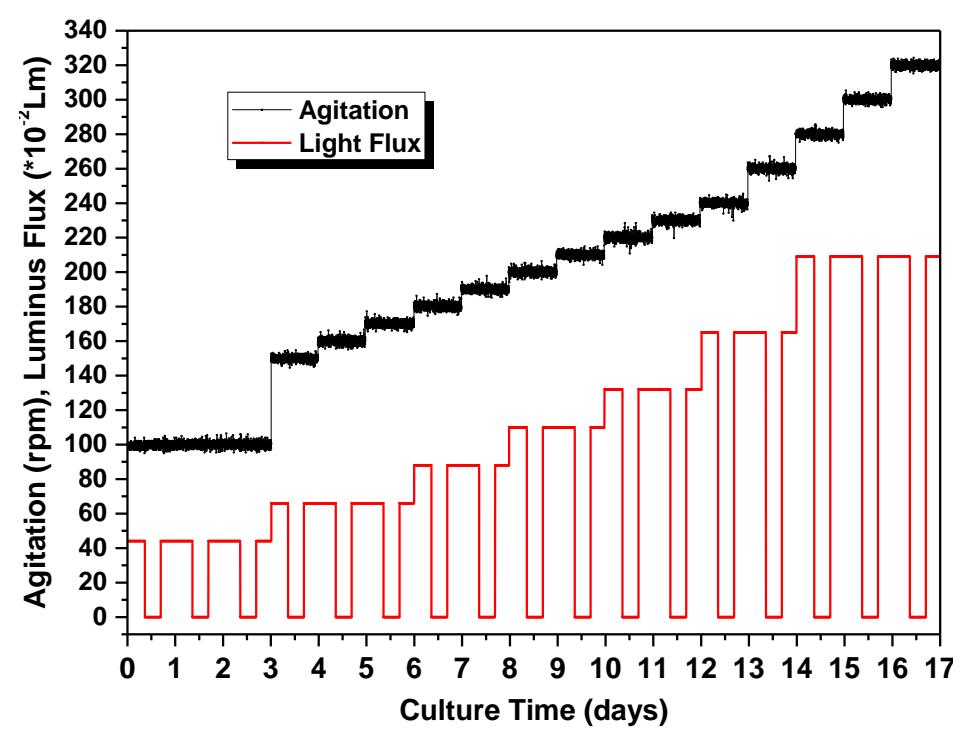

Figure 3: Time - escalating profile of illumination and agitation rate applied in the experiments WL, WRL, and WBL.

Three independent batch experiments were conducted in a bench-top photobioreactor in two replicates (mean values are reported). In the WL experiment, white lighting was exclusively applied throughout the 17-day cultivation period. The culture started with only four lamps on $(4400 \mathrm{~lm})$, and successively two $(2200 \mathrm{~lm})$ or three additional LED lamps $(3300 \mathrm{~lm})$ were set on at predefined time instants. In the WRL experiment, white and red lighting was applied throughout the 17-day cultivation period. The time schedule for the white lighting was identical to the WL, however the culture started with the red-light band being reinforced by five red LED strips. Finally, in the WBL experiment, white and blue lighting was applied throughout the 17-day cultivation period; the time schedule employed for the white and blue illumination was the same as the one imposed in the WRL. In Table 2, the initial and final lighting conditions in 
all three experiments are reported, while the respective dynamic profiles are shown jointly with the agitation profile in Figure 3 and discussed in the following section. Thus, it was expected that sufficient exposure of the cells to light was ensured, and the inhibitory effect of the cells' self-shading phenomenon was confined.

Table 2: Light conditions of the experimental study.

\begin{tabular}{|l|l|l|l|}
\hline Experiment & Light Conditions & $\begin{array}{l}\text { Luminous Flux at } \\
\text { the First Day } \\
(\mathbf{L m})\end{array}$ & $\begin{array}{l}\text { Luminous Flux at } \\
\text { the Final Day } \\
\text { (Lm) }\end{array}$ \\
\hline WL & White light & 4400 & 20,900 \\
\hline WRL & White and red light & 5975 & 22,475 \\
\hline WBL & $\begin{array}{l}\text { White and blue } \\
\text { light }\end{array}$ & 4925 & 21,425 \\
\hline
\end{tabular}

\section{Results and Discussion Evaluation of the Photosynthetic Activity}

Prior to the quantitative assessment of the light spectrum impact on the culture growth, it is worthy to preliminary explore, through macroscopic process indexes, how and whether the intended incremental lighting policies sufficiently supported the photosynthetic activity of the cellular population along the 17-d cultivation period, using primarily the WL experiment as a reference case. The culture response to the applied photoperiod $(16: 8 \mathrm{~h})$ was evident, particularly through the immediate change of the culture $\mathrm{pH}$ (Figure 4a) and the composition variance in the outflow gas stream (Figure 4b), both synchronized with the imposed dark-light alternation. 
Prime Archives in Sustainability

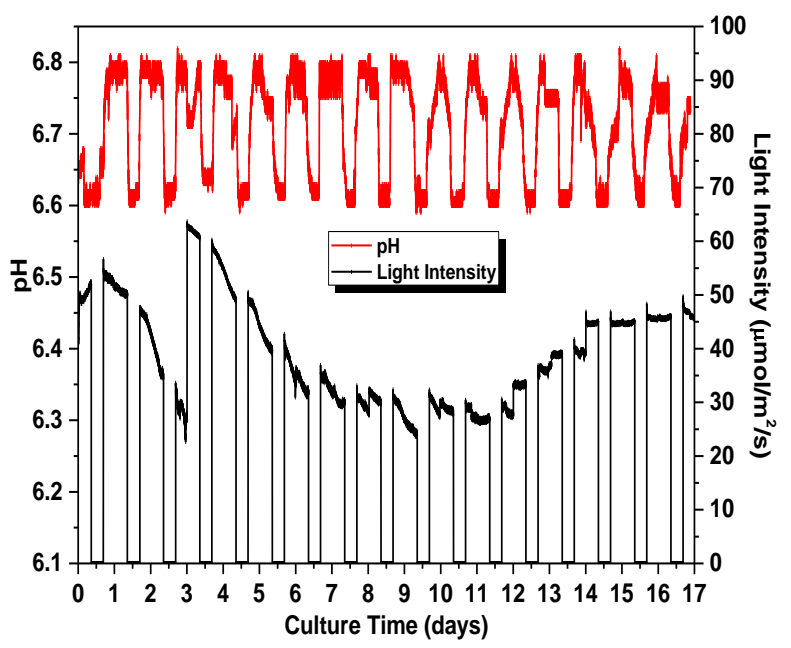

(a)

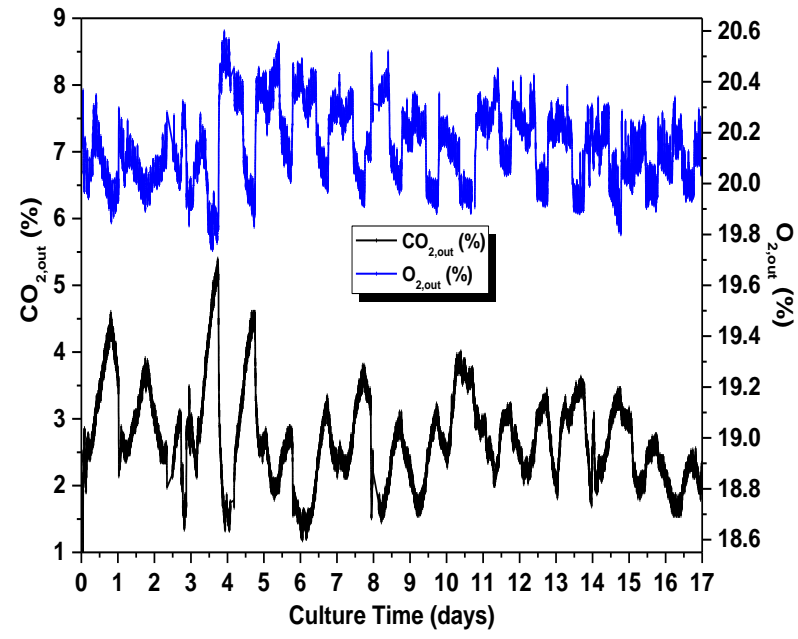

(b)

Figure 4: Time profile of process response under White Light (WL) during the 17-d culture period in terms of: (a) $\mathrm{pH}$ and-light intensity (measured by the sensor submerged inside the culture) and, (b) outflow gas stream composition (\% $\mathrm{mol} / \mathrm{mol})$. 
Precisely, with the onset of illumination, an immediate increase in the value of $\mathrm{pH}$ was recorded. It is known that during light reactions in the cycle of photosynthesis, microalgae consume carbon dioxide; this was verified by the $\mathrm{pH}$ increase up to the maximum allowable value (i.e., 6.8) during the lighting period, while in the dark reactions the opposite process occurred, resulting in the minimum allowable $\mathrm{pH}$ value (i.e., 6.6). Similar analysis over the light effect on the outflow gas stream composition showed that at the beginning of each lighting period an increase in the $\mathrm{O}_{2}$ molar fraction in the gaseous stream and a decrease in the respective $\mathrm{CO}_{2}$ fraction was observed. By the end of the illumination period, the exact reverse behavior was recorded (Figure $4 b$ ).

Furthermore, it is worthy to commend that at the start of each light cycle, the light intensity inside the reactor, where the light sensor is immersed, got the highest value for each cycle and then it was gradually reduced along the 16-h lighting period (black line in Figure 4a). This is clear evidence of the intense photosynthetic activity, further confirmed by the respective OD measurements. However, as depicted in Figure 4a, from the 13th day of cultivation onwards, it was no more sharp decreases of the measured light intensity inside the PBR were observed, which remained almost stable instead. This indicates a lower photosynthetic activity and a slower growth rate during the lighting period, while it might also imply a steadiness in cell content in total pigments. Similar behavior was observed in the other two experiments, and although the respective data for $\mathrm{pH}$ and $\mathrm{O}_{2}-\mathrm{CO}_{2}$ molar fractions are not presented, the time profile of the light intensity at the fixed point within the culture medium is commendable and the comparison of this time trajectory for the three experiments is presented in Figure 5.

Note that despite the extra culture illumination with red (WRL) or blue light (WBL), the respective intensity at the culture internal point was further reduced compared to the WL experiment. Although such behavior might be explained by a respective culture optical density or/and DCW increase, it will be proven next that it was induced by the contained pigments in the cells, as will be discussed in Section 4.3. 


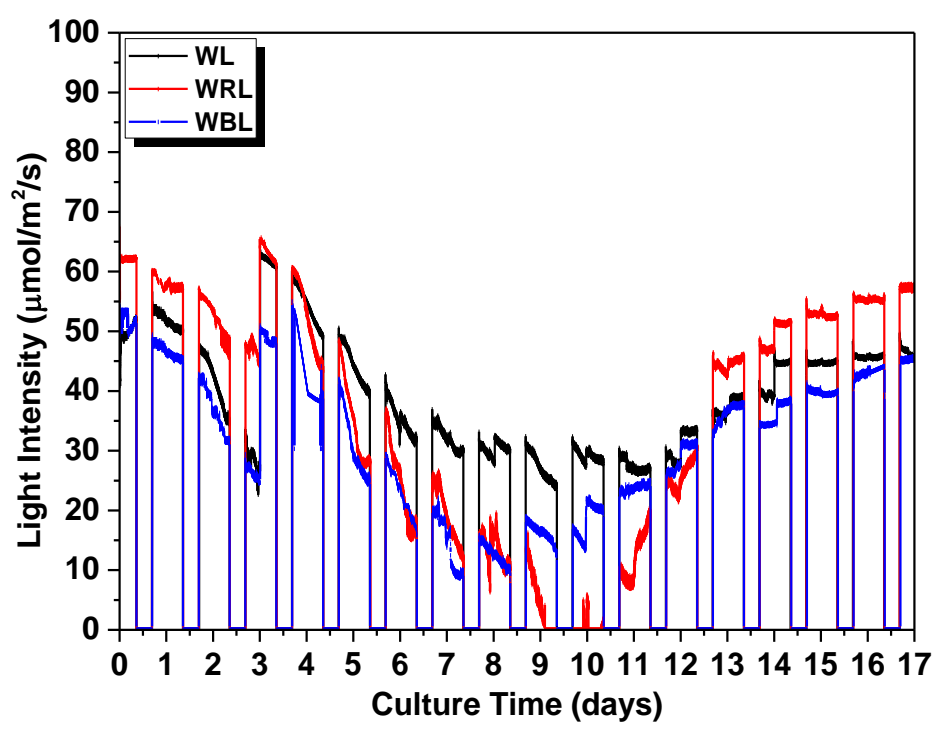

Figure 5: Light intensity profiles measured inside the culture medium during the experiments WL, WRL, and WBL.

\section{Mixing and Lighting Profile Effect on OD and DCW}

The concept of the progressively increasing incident light flux combined with the rising agitation rate of the culture was a dual purpose decision and was expected to: (i) satisfy the consecutively expanded culture light energy requirements due to the cell population growth, and (ii) sustain simultaneous homogeneity and sufficiency in the exposure time of cells to the light radiation and excitation of their photosynthetic centers due to the formation of darker layers radially from the surface to the center of the bioreactor. This concept was actually incarnated by the heuristically designed strategy shown in Figure 3, and its effectiveness is demonstrated by the dynamic profile of the Stichococcus sp. culture growth, expressed either by the OD or the dry cell mass concentration (DCW), for the three experiments, as shown in Figure 6a and Figure 6b. Provision of light energy depending on the culture needs and more intensive mixing proportionally to the culture density resulted in the elongation of the exponential growth period to 14 days or more in all three experiments. 
Elaborating on the effect of the light type, it was unambiguously concluded that the specific wavelength indeed affects Stichococcus sp. growth. When the culture was exposed exclusively to white light it reached the maximum OD value compared to the experiments where white and red or white and blue lights were combined, despite the fact that the respective OD time trajectories were almost identical for the first 13 days in the three experiments (Figure 6a). Consistent with the OD graph, the respective profiles of DCW in the three experiments indicate that the culture in the WL experiment reached the highest DCW value (i.e., $3.5 \mathrm{~g} / \mathrm{L}$ ) at the end of the $17-\mathrm{d}$ cultivation period (Figure 6b). Notice though that under the three lighting policies the course of the culture growth changed despite the resemblance of the OD trajectories in the respective experiments for the first 13 days. While in the WRL and WBL experiments the cultures entered from the third day in a phase of exponential growth that lasted until the 12th day, in the WL the exponential growth phase started on the 8th day and the culture exhibited aggressive growth almost until the end (measurements on the 18th day showed a DCW value of $3.7 \mathrm{~g} / \mathrm{L}$, indicating that the culture completed the exponential growth phase and started the static phase - these values were not included in Figure 6b). The results of the present work are consistent with various other published studies [29,30,44] investigating the effect of different light wavelengths on various microalgae strains. 
Prime Archives in Sustainability

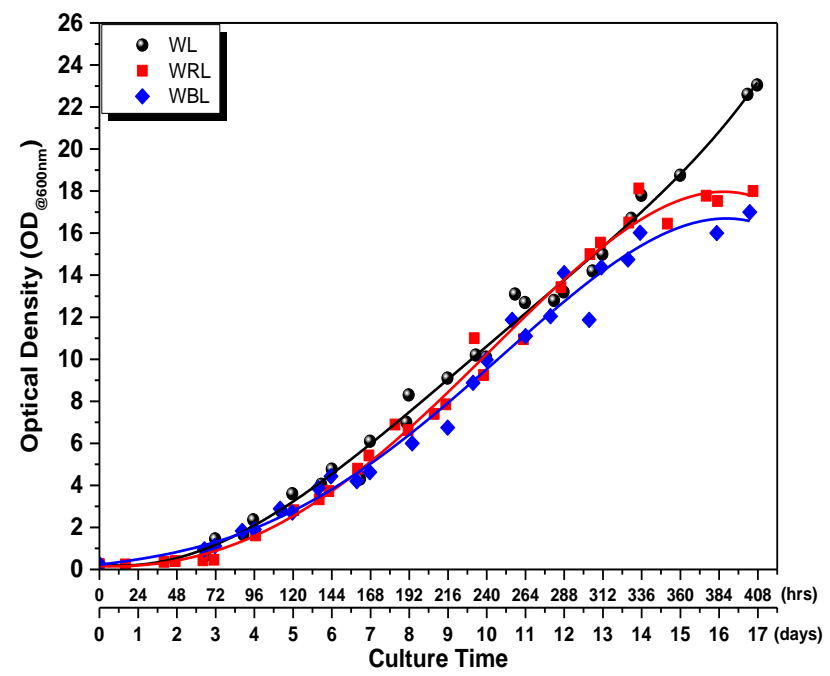

(a)

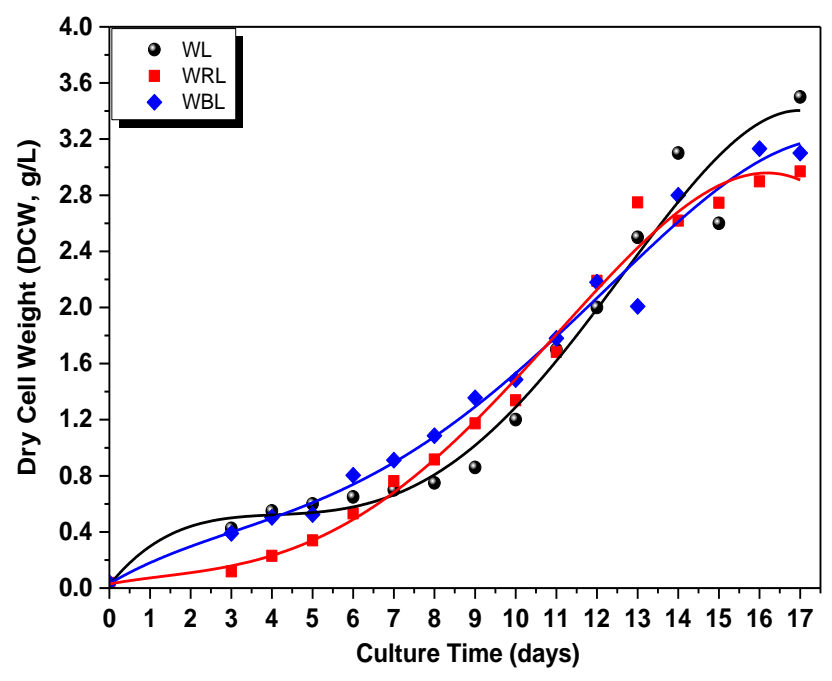

(b)

Figure 6: Culture response to different illumination policies in terms of: (a) Optical Density $\left(\mathrm{OD}_{600 \mathrm{~nm}}\right)$ and (b) Dry Cell Weight.

In contrast to these results, a recent study on S.bacillaris alleged that the use of blue or red light did not seriously affect the 
culture growth kinetics [31], and yet several other published articles stated that red light is commonly suggested for biomass productivity enhancement in green microalgae [14, 24-27]. This is justified on the basis of the concurrence of the absorbance area of chlorophylls $\mathrm{a}$ and $\mathrm{b}$ (present in the light-harvesting complexes of green algae) with the red light spectrum. There are several factors that can explain the discrepancy of the present work with the above statements, including primarily: (i) the fact that red and blue light are applied separately in most studies and not in addition to the white light spectrum, as implemented by the present work; (ii) the use of different microalgae species, reflecting serious differences in cellular structure, pigment and photoreceptor composition, and chloroplast arrangement; as well as (iii) the inconsistency of the red and blue spectrum used in the present study (the employed stipes emit in 630/460 nm, respectively) with the corresponding light sources employed by other researchers (e.g., emission in 660/390-570 nm. [31]). In order to further distinguish the role of different light conditions on the cellular composition of the studied Stichococcus sp. cultures, in combination with their respective performance, the dynamic profile of the culture growth was monitored and analyzed in parallel with the time trajectories of the concentrations of the products of interest during the 17-day cultivation period.

\section{Metabolites Concentration}

The additional luminous energy provided in the WRL and WBL experiments would be expected to lead to a higher biomass concentration than in the WL one. However, exposure of the culture to extra red or blue, in addition to white, light appeared to activate metabolic mechanisms of component accumulation and simultaneous restriction of the proliferation of the cell population. However, the two types of lighting (white-red light combination and white-blue light combination) seem to enhance different metabolic pathways.

In particular, the culture illumination exclusively with white light led to a significantly higher final concentration of carbohydrates (i.e., $1.15 \mathrm{~g} / \mathrm{L}$ ) compared to the combined forms 
of lighting (Figure 7a). Besides, from all three experiments, it is understood that the time evolution of carbohydrate synthesis and accumulation goes in parallel with the corresponding biomass concentration time trajectory. Thus, the effect of the type of lighting on this intracellular metabolite conforms to the general effect it has on the culture growth. If one considers that the carbohydrate accumulation by a cell population serves as a direct access energy store, it makes sense to match the carbohydrate concentration profile with the corresponding biomass profile. This consequently explains the enhancement of the carbohydrate synthesis mechanism by the policy of exclusively white lighting that also enhances the dry cell mass production. This relevance of the carbohydrate accumulation in Stichococcus sp. cultures with the increase of dry biomass concentration was additional recorded by a previous study [32].

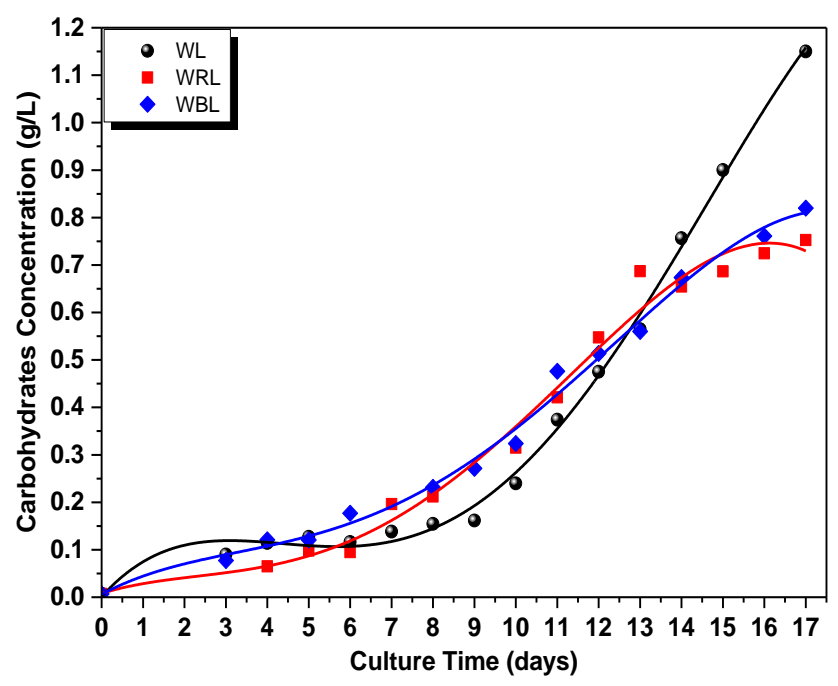

(a) 
Prime Archives in Sustainability

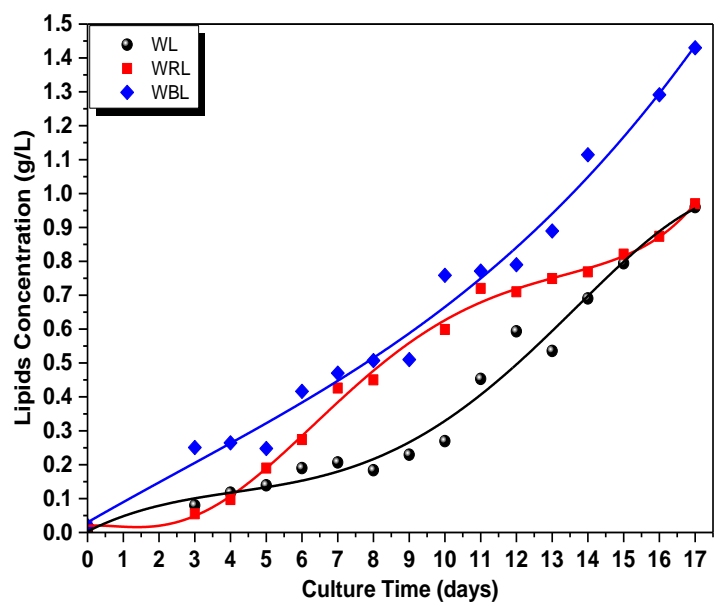

(b)

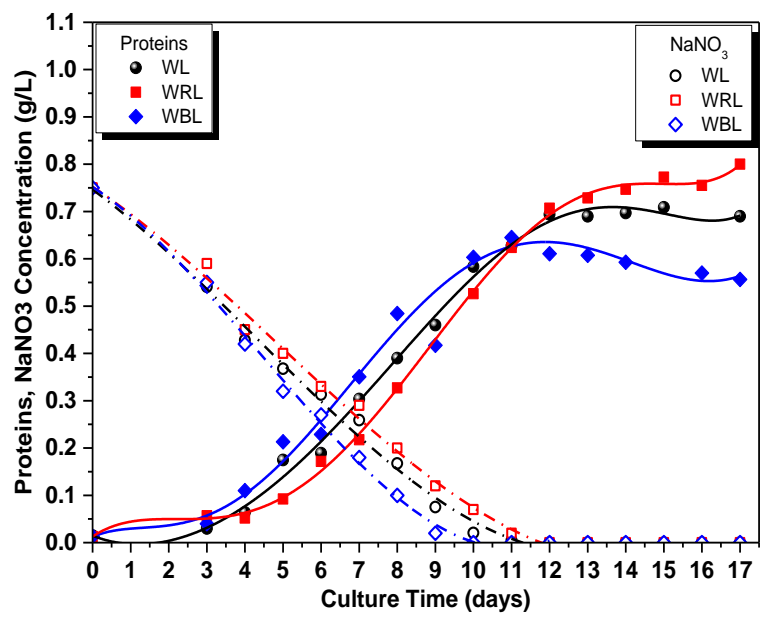

(c)

Figure 7: Time profile of products and nitrogen concentration during the experiments WL, WRL, and WBL: (a) Carbohydrates concentration, (b) Lipids concentration, (c) Proteins and nitrogen source $\left(\mathrm{NaNO}_{3}\right)$ concentration.

Regarding the other two products, however, there seems to be a selective amplification of their individual synthesis by the lighting type. More specificly, the combination of white-blue lighting had a favoring effect on the profile of the lipids 
concentration in the three experiments compared to the other two types of lighting (Figure 7b). This is clearly demonstrated by the uninterrupted increase of accumulated lipids from the beginning of the respective culture (WBL), resulting in the exceptionally high final concentration and content value of $1.43 \mathrm{~g} / \mathrm{L}$ and $46.13 \%$ wt., respectively. This experimental observation is further supported by various research studies reporting that, under blue light, microalgae tend to maximize their intracellular lipid content $[22,23,30]$. Such behavior can be attributed to the fact that blue light affects the metabolic pathway of fatty acid formation by stimulating specific enzymes. Precisely, the ribulose-1,5-bisphosphate carboxylase/oxygenase (Rubisco), being the most critical enzyme in the Calvin-Benson (CB) cycle that involves thirteen different enzymatic reactions, is a key factor for controlling carbon fixation [18]. According to Roscher and Zetsche (1986), who studied the effect of light on this enzyme, blue light of a wavelength ranging from 430 to $510 \mathrm{~nm}$, with a peak at about $460 \mathrm{~nm}$, causes an increase in RuBisCo synthesis [45]. Thus, the cool white light enhancement with blue light $(460 \mathrm{~nm})$, implemented in the present study, had a very distinct impact on the dynamic profile of lipids accumulation by Stichococcus sp. cells and resulted in the highest lipid concentration.

It is clear that no matter the type of lighting, protein synthesis requires nitrogen sufficiency. Measurements performed at $24 \mathrm{~h}$ intervals indicate that the nitrogen contained in the medium $(0.75$ $\mathrm{g} / \mathrm{L} \mathrm{NaNO}_{3}$ ) was depleted within approximately 9-11 days of the culture in all three experiments (Figure 7c). This deficiency of the nitrogen source in the medium resulted in an evident inhibition on the synthesis of proteins and a consequent static and slightly decreasing trend of their concentration profile, as shown in Figure $7 \mathrm{c}$ for the three experiments. However, it can be concluded that the white-red light combination regulates the metabolism of the cell population towards the selective utilization of the supplied nitrogen $\left(\mathrm{NO}_{3}{ }^{-}\right)$-to-protein synthesis to manifest in the respective culture the highest concentration of proteins $(0.8 \mathrm{~g} / \mathrm{L})$, but at the same time a milder rate of cell proliferation and the lowest final concentration of dry biomass $(2.97 \mathrm{~g} / \mathrm{L})$. Remarkable is the fact that under the combination of 
white-blue lighting the lower final protein concentration is achieved $(0.56 \mathrm{~g} / \mathrm{L})$, since under this type of lighting the metabolic pathway of lipid synthesis is more prevalent.

The bar chart in Figure 8 records the weight composition of dry biomass concerning products of interest (i.e., carbohydrates, lipids, proteins) in the three experiments of different types of lighting. With reference to the WL experiment, it was concluded that the combination of white and red lighting and yet more white and blue lighting contributes to the beneficial utilization of light and nutrients by increasing the final composition of dry biomass in total products while reducing the residual biomass (mainly consisting of minerals, dietary fibers, pigments, and ash $[46,47])$.

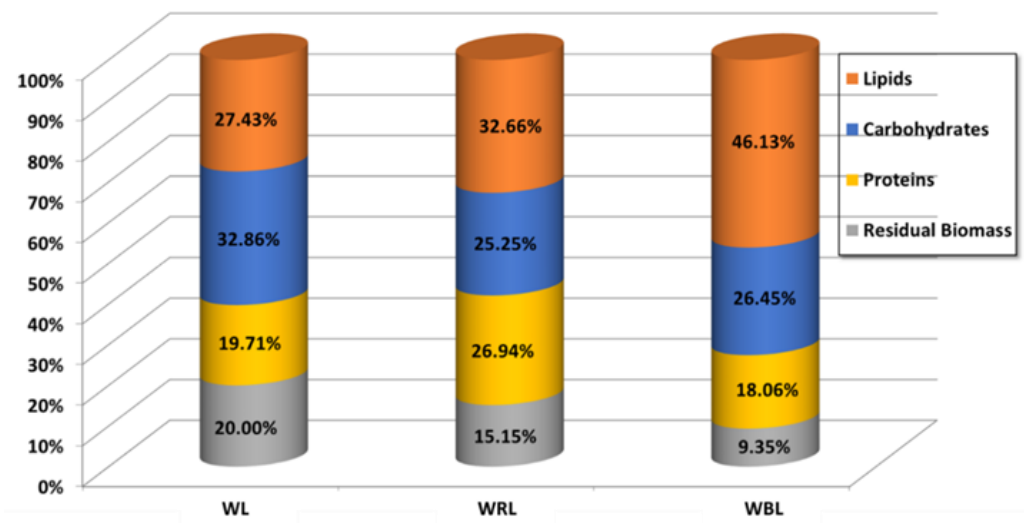

Figure 8: Biomass composition of Stichococcus sp. cells $(\% \mathrm{~g} / \mathrm{g})$ at the end of the 17-day cultivation period under the conditions of the experiments WL, WRL, and WBL.

Finally, it was confirmed that the amplification of white light in the red band guides the photosynthetic utilization of $\mathrm{CO}_{2}$ towards the synthesis of proteins, increasing the final composition of biomass in proteins from $19.71 \%$ to $26.94 \%$ wt. On the other hand, the amplification of white light in the blue band significantly intensifies the photosynthetic utilization of $\mathrm{CO}_{2}$ towards lipid synthesis by increasing the final composition of biomass in lipids from $27.43 \%$ to $46.13 \%$ wt. 
In summary, the policy of exclusively white lighting results in the maximum concentration of carbohydrates but leads to moderate final concentrations of the other two products (lipids and proteins) (see Table 3). Lipid concentration is maximized when white light is combined with blue and this is to the detriment of both carbohydrates and proteins (comparison of final carbohydrate and protein concentrations in WL vs. WBL experiments). Respectively, the concentration of proteins is maximized when the white light is combined with red and this is done exclusively at the expense of carbohydrates, since the final concentration of lipids in the WL vs. WRL experiment was almost identical (0.96 and $0.97 \mathrm{~g} / \mathrm{L}$, respectively).

Table 3: Biomass composition determined at the end of the 17-day experimental period of Stichococcus sp. cultures with different illumination policies.

\begin{tabular}{|l|l|l|l|l|}
\hline Experiment & $\begin{array}{l}\text { Biomass } \\
\text { Concentr } \\
\text { ation } \\
\text { (g/L) }\end{array}$ & $\begin{array}{l}\text { Protein } \\
\text { Concentra } \\
\text { tion }(\mathrm{g} / \mathrm{L})\end{array}$ & $\begin{array}{l}\text { Lipid } \\
\text { Concentra } \\
\text { tion }(\mathrm{g} / \mathrm{L})\end{array}$ & $\begin{array}{l}\text { Carbohydrates } \\
\text { Concentration } \\
\text { (g/L) }\end{array}$ \\
\hline WL & 3.5 & 0.69 & 0.96 & 1.15 \\
\hline WRL & 2.97 & 0.8 & 0.97 & 0.75 \\
\hline WBL & 3.1 & 0.56 & 1.43 & 0.82 \\
\hline
\end{tabular}

Comparing the results of the WL experiment with those of the other two experiments (WRL and WBL), the lower concentration of chlorophylls $a$ and $b$ during the entire cultivation period is remarkable (Figure 9). These findings are explicitly correlated with the experimental indications of the light intensity provided by the sensor inside the culture volume (Figure 5). Even if, under the WRL and WBL experiment, the incident light flux was higher than in the WL experiment (see Table 2) and the WL experiment resulted in higher OD values compared to the WRL and WBL experiment, the light intensity inside the PBR exhibited higher values during the WL illumination policy. The higher content of the respective cells in pigments in the WRL and WBL experiments restrained the light penetration inside the culture, resulting in the lower intensity values that were recorded. 


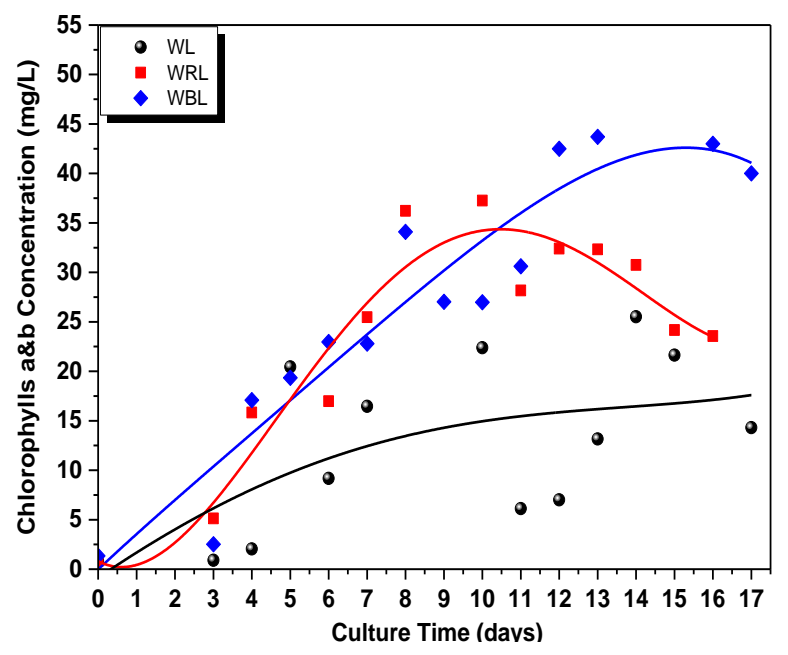

Figure 9: Time profile of chlorophylls concentration during the experiments WL, WRL, and WBL.

\section{Conclusions}

The microalgae photosynthetic cultivation process, combining cell proliferation and biomass production and intracellular accumulation of particular products, turned out to be seriously dependent on the quality of available light in the culture. Thus, the utilization of suitable wavelengths of visible light could prove to be an effective strategy to guide microalgae cultures, targeting the overexpression of the desired intracellular metabolites. However, the decision over the level of exposure of the culture to the employed light by cautious selection of the luminous flux and culture mixing time profiles, in combination with the $\mathrm{CO}_{2}$ feeding, can only be considered as an integral part of such a strategy.

Stichococcus sp. could be successfully used as a potential cell factory for an integrated biorefinery plant, considering its ability to create high-density cell populations and efficiently accumulate three prominent metabolites, carbohydrates, proteins, and lipids. Using different light wavelengths on the cultivation of Stichococcus sp. can contribute to the directed photosynthetic production of different useful products, in conformity with the 
operational framework desired in a biorefinery plant. White LED light has been proved to favor the Stichococcus sp. culture growth up to large dry cell mass values, while it has been immediately associated with the intensive accumulation of carbohydrates by the cells. On the other hand, enhancing the white light spectrum with blue LED light triggers the overproduction of lipids, favoring the assimilation of $\mathrm{CO}_{2^{-}}$ carbon preferentially in the metabolic pathway of lipid synthesis, while the white light enhancement in the red band is a promising strategy to intensify the accumulation of proteins when sufficient nitrogen supply is ensured. Finally, this work indicated that both red and blue light in combination with white can dramatically increase chlorophylls concentrations.

The knowledge generated in the present research is expected to support the efficient scaling-up of the microalgae cultivation process, which is of primary importance for the development and establishment of a potential microalgae-based technology.

\section{References}

1. Efroymson RA, Jager HI, Mandal S, Parish ES, Mathews TJ. Better Management Practices for Environmentally Sustainable Production of Microalgae and Algal Biofuels. J. Clean. Prod. 2021; 289: 125150.

2. Chisti Y. Biodiesel from Microalgae. Biotechnol. Adv. 2007; 25: 294-306.

3. Ahmad AL, Yasin NHM, Derek CJC, Lim JK. Microalgae as a Sustainable Energy Source for Biodiesel Production: A Review. Renew. Sustain. Energy Rev. 2011; 15: 584-593.

4. Lam GP, Vermuë MH, Eppink MHM, Wijffels RH, van den Berg C. Multi-Product Microalgae Biorefineries: From Concept Towards Reality. Trends Biotechnol. 2018; 36: 216-227.

5. Laurens LML, Markham J, Templeton DW, Christensen ED, Van Wychen S, et al. Development of Algae Biorefinery Concepts for Biofuels and Bioproducts, a Perspective on Process-Compatible Products and Their Impact on CostReduction. Energy Environ. Sci. 2017; 10: 1716-1738. 
6. Adams C, Godfrey V, Wahlen B, Seefeldt L, Bugbee B. Understanding Precision Nitrogen Stress to Optimize the Growth and Lipid Content Tradeoff in Oleaginous Green Microalgae. Bioresour. Technol. 2013; 131: 188-194.

7. Saha SK, McHugh E, Hayes J, Moane S, Walsh D, et al. Effect of Various Stress-Regulatory Factors on Biomass and Lipid Production in Microalga Haematococcus pluvialis. Bioresour. Technol. 2013; 128: 118-124.

8. Pérez-Pazos JV, Fernández-Izquierdo P. Synthesis of Neutral Lipids in Chlorella sp. under Different Light and Carbonate Conditions. CTF Cienc. Tecnol. Futuro 2011; 4: 47-57.

9. Olivieri G, Gargano I, Andreozzi R, Marotta A, Marzocchella A, et al. Effects of $\mathrm{CO}_{2}$ and $\mathrm{pH}$ on Stichococcus bacillaris in Laboratory Scale Photobioreactors, Chemical Engineering Transactions. Milano: AIDIC. 2012; 27.

10. Roleda MY. Effects of Temperature and Nutrient Regimes on Biomass and Lipid Production by Six Oleaginous Microalgae in Batch Culture Employing a Two-Phase Cultivation Strategy. Bioresour. Technol. 2013; 11: 439449.

11. de Castro Araújo S, Garcia VMT. Growth and Biochemical Composition of the Diatom Chaetoceros Cf. Wighamii Brightwell under Different Temperature, Salinity and Carbon Dioxide Levels. I. Protein, Carbohydrates and Lipids. Aquaculture. 2005; 246: 405-412.

12. Aguilera J, Gordillo L, Karsten U, Figueroa FL, Niell FX. Light Quality Effect on Photosynthesis and Efficiency of Carbon Assimilation in the Red Alga Porphyra Leucosticta. J. Plant Physiol. 2000; 157: 86-96.

13. Korbee N, Figueroa FL, Aguilera J. Effect of Light Quality on the Accumulation of Photosynthetic Pigments, Proteins and Mycosporine-like Amino Acids in the Red Alga Porphyra Leucosticta (Bangiales, Rhodophyta). J. Photochem. Photobiol. B 2005; 80: 71-78.

14. Kim DG, Lee C, Park SM, Choi YE. Manipulation of Light Wavelength at Appropriate Growth Stage to Enhance Biomass Productivity and Fatty Acid Methyl Ester Yield 
Using Chlorella vulgaris. Bioresour. Technol. 2014; 159: 240-248.

15. Adholeya A. A Review on the Assessment of Stress Conditions for Simultaneous Production of Microalgal Lipids and Carotenoids. Front. Microbiol. 2016; 7: 19.

16. Carvalho AP, Silva SO, Baptista JM, Malcata FX. Light Requirements in Microalgal Photobioreactors: An Overview of Biophotonic Aspects. Appl. Microbiol. Biotechnol. 2011; 89: $1275-1288$.

17. Kommareddy A. Study of Light as a Parameter in the Growth of Algae in a Photo-Bio Reactor (PBR). In Proceedings of the 2003 ASAE Annual Meeting. Las Vegas, NV, USA. 2003.

18. Ooms MD, Dinh CT, Sargent EH, Sinton D. Photon Management for Augmented Photosynthesis. Nat. Commun. 2016; 7: 12699.

19. Ramanna L, Rawat I, Bux F. Light Enhancement Strategies Improve Microalgal Biomass Productivity. Renew. Sustain. Energy Rev. 2017; 80: 765-773.

20. Masojídek J, Torzillo G, Koblížek M. Photosynthesis in Microalgae. In: Richmond A, Hu Q, editors. Handbook of Microalgal Culture. Oxford: John Wiley \& Sons, Ltd. 2013; 21-36.

21. Schulze PSC. Light Emitting Diodes (LEDs) Applied to Microalgal Production. Trends Biotechnol. 2014; 32: 422 430.

22. Teo CL, Atta M, Bukhari A, Taisir M, Yusuf AM, et al. Enhancing Growth and Lipid Production of Marine Microalgae for Biodiesel Production via the Use of Different LED Wavelengths. Bioresour. Technol. 2014; 162: 38-44.

23. Das P, Lei W, Aziz SS, Obbard JP. Enhanced Algae Growth in Both Phototrophic and Mixotrophic Culture under Blue Light. Bioresour. Technol. 2011; 102: 3883-3887.

24. Kendirlioglu G, Cetin AK. Effect Of Different Wavelengths Of Light On Growth, Pigment Content And Protein Amount Of Chlorella vulgaris. Fresenius Environ. Bull. 2017; 26: 7974-7980.

25. Shu CH, Tsai CC, Liao WH, Chen KY, Huang HC. Effects of Light Quality on the Accumulation of Oil in a Mixed 
Culture of Chlorella sp. and Saccharomyces Cerevisiae. J. Chem. Technol. Biotechnol. 2012; 87: 601-607.

26. Oldenhof HT, Zachleder VM. Blue- and Red-Light Regulation of the Cell Cycle in Chlamydomonas reinhardtii (Chlorophyta). Eur. J. Phycol. 2006; 41: 313-320.

27. Koc C, Anderson GA, Kommareddy A. Use of Red and Blue Light-Emitting Diodes (LED) and Fluorescent Lamps to Grow Microalgae in a Photobioreactor. Isr. J. Aquacult. Bamid. 2013; 65: 797-804.

28. Rendón SM, Roldan GJC, Voroney RP. Effect of Carbon Dioxide Concentration on the Growth Response of Chlorella Vulgaris Under Four Different Led Illumination. Int. J. Biotechnol. Wellness Ind. 2013; 2: 125-131.

29. Kim TH, Lee Y, Han SH, Hwang SJ. The Effects of Wavelength and Wavelength Mixing Ratios on Microalgae Growth and Nitrogen, Phosphorus Removal Using Scenedesmus sp. for Wastewater Treatment. Bioresour. Technol. 2013; 130: 75-80.

30. Abiusi F, Sampietro G, Marturano G, Biondi N, Rodolfi L, et al. Growth, Photosynthetic Efficiency, and Biochemical Composition of Tetraselmis Suecica F\&M-M33 Grown with LEDs of Different Colors: Growth of Tetraselmis With Different LED Colors. Biotechnol. Bioeng. 2014; 111: 956964.

31. Mutaf T, Oz Y, Kose A, Elibol M, Oncel SS. The Effect of Medium and Light Wavelength towards Stichococcus bacillaris Fatty Acid Production and Composition. Bioresour. Technol. 2019; 289: 121732.

32. Karapatsia A, Penloglou G, Chatzidoukas C, Kiparissides C. An Experimental Investigation of Stichococcus sp. Cultivation Conditions for Optimal Co-Production of Carbohydrates, Proteins and Lipids Following a Biorefinery Concept. Biomass Bioenergy. 2016; 89: 123-132.

33. Brown LM, Hellebust JA. Some New Taxonomic Characteristics Applied to Stichococcus Bacillaris (Chlorophyceae). Can. J. Bot. 1980; 58: 1405-1411.

34. Hughes KA. Solar UV-B Radiation, Associated with Ozone Depletion, Inhibits the Antarctic Terrestrial Microalga, Stichococcus Bacillaris. Polar Biol. 2006; 29: 327-336. 
35. Sheehan J, Dunahay T, Benemann J, Roessler P. Look Back at the U.S. Department of Energy's Aquatic Species Program: Biodiesel from Algae, Close-Out Report, NREL/TP-580-24190. National Renewable Energy Lab. Golden, CO.(US). 1998; 15003040.

36. Zhang Weixing, Majidi, Vahid. Monitoring the Cellular Response of Stichococcus Bacillaris to Exposure of Several Different Metals Using in Vivo 31P NMR and Other Spectroscopic Techniques. Environ. Sci. Technol. 1994; 28: $1577-1581$.

37. Wellburn AR. The Spectral Determination of Chlorophylls a and b, as Well as Total Carotenoids, Using Various Solvents with Spectrophotometers of Different Resolution. J. Plant Physiol. 1994; 144: 307-313.

38. Henriques M, Silva A, Rocha J. Extraction and Quantification of Pigments from a Marine Microalga: A Simple and Reproducible Method. Communic. Current Res. Educ. Topics Trends Appl. Microbiol. 2007; 2: 586-593.

39. Folch J, Lees M, Sloane Stanley GH. A Simple Method for the Isolation and Purification of Total Lipides from Animal Tissues. J. Biol. Clemistry. 1956; 226: 497-509.

40. Chen Y, Vaidyanathan S. Simultaneous Assay of Pigments, Carbohydrates, Proteins and Lipids in Microalgae. Anal. Chim. Acta. 2013; 776: 31-40.

41. Schwenzfeier A, Wierenga PA, Gruppen H. Isolation and Characterization of Soluble Protein from the Green Microalgae Tetraselmis sp. Bioresour. Technol. 2011; 102: 9121-9127.

42. DuBois Michel, Gilles KA, Hamilton JK, Rebers PA, Smith Fred. Colorimetric Method for Determination of Sugars and Related Substances. Anal. Chem. 1956; 28: 350-356.

43. Collos Y, Mornet F, Sciandra A, Waser N, Larson A, et al. An Optical Method for the Rapid Measurement of Micromolar Concentrations of Nitrate in Marine Phytoplankton Cultures. J. Appl. Phycol. 1999; 11: 179-184.

44. Wong Y, Ho YH, Ho, kin C, Leung HM, et al. Effect of Different Light Sources on Algal Biomass and Lipid Production in Internal Leds-Illuminated Photobioreactor. J. Mar. Biol. Aquac. 2016; 2: 1-8. 
45. Roscher E, Zetsche K. The Effects of Light Quality and Intensity on the Synthesis of Ribulose-1,5-Bisphosphate Carboxylase and Its MRNAs in the Green Alga Chlorogonium Elongatum. Planta. 1986; 167: 582-586.

46. Matos ÂP, Feller R, Moecke EHS, de Oliveira JV, Junior AF, et al. Chemical Characterization of Six Microalgae with Potential Utility for Food Application. J. Am. Oil Chem. Soc. 2016; 93: 963-972.

47. Molino A, Iovine A, Casella P, Mehariya S, Chianese S, et al. Microalgae Characterization for Consolidated and New Application in Human Food, Animal Feed and Nutraceuticals. Int. J. Environ. Res. Public. Health. 2018; 15: 2436. 